\title{
Principles of Verifiable RTL Design Second Edition
}




\section{Principles of Verifiable RTL Design}

Second Edition

A functional coding style supporting verification processes in Verilog

Lionel Bening and Harry Foster Hewlett-Packard Company 
eBook ISBN: $\quad 0-306-47631-2$

Print ISBN: $\quad$ 0-7923-7368-5

(C2002 Kluwer Academic Publishers

New York, Boston, Dordrecht, London, Moscow

Print (C2001 Kluwer Academic Publishers

Dordrecht

All rights reserved

No part of this eBook may be reproduced or transmitted in any form or by any means, electronic, mechanical, recording, or otherwise, without written consent from the Publisher

Created in the United States of America

Visit Kluwer Online at:

http://kluweronline.com

and Kluwer's eBookstore at:

http://ebooks.kluweronline.com 
Dedicated to:

Ann, Laura and Donna

-Lionel

Roger, Elliott, Lance, Hannah and Jeanne

-Harry 


\section{Table of Contents}

\section{FOREWORD}

XV

\section{PREFACE} xix

\section{Introduction} 1

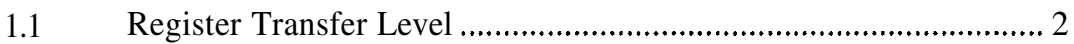

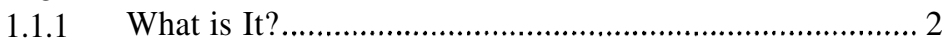

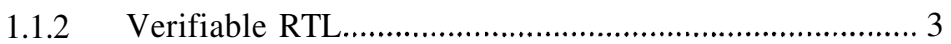

1.1.3 Applying Design Discipline ............................................ 4

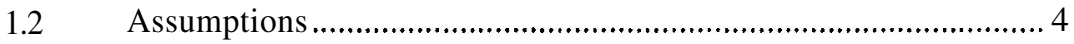

$1.3 \quad$ Organization of This Book......................................................... 5

2 The Verification Process .............................................9

$2.1 \quad$ Specification Design Decomposition ........................................... 10

2.1.1 High-Level Design Requirements ................................... 11

2.1.2 Block-Level Specification and Design ............................ 13

2.1.3 RTL Implementation ................................................... 13

2.1.4 Synthesis and Physical Design .................................... 13

2.2 Functional Test Strategies ........................................................ 14

2.2.1 Deterministic or Directed Test........................................ 15

2.2.2 Random Test................................................................... 16

2.2.3 Transaction Analyzer Verification ................................. 18

2.2.4 Chip Initialization Verification ....................................... 19

2.2.5 Synthesizable Testbench.................................................... 20 


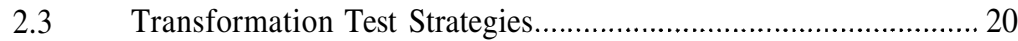

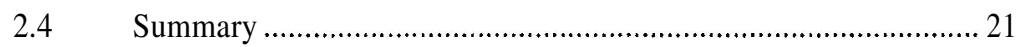

3 Coverage, Events and Assertions .......................................23

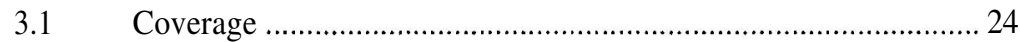

3.1.1 Ad-hoc Metrics ....................................................... 25

3.1.2 Programming Code Metrics.......................................... 25

3.1.3 State Machine and Arc Coverage Metrics ..................... 27

3.1.4 User Defined Metrics................................................... 27

3.1.5 Fault Coverage Metrics............................................... 27

3.1.6 Regression Analysis and Test Suite Optimization........... 28

3.2 Event Monitors and Assertion Checkers....................................... 28

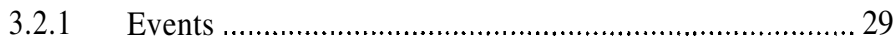

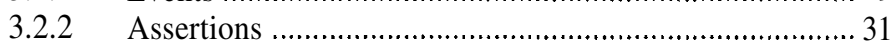

3.2.3 Assertion Monitor Library Details................................. 36

3.2.4 Event Monitor and Assertion Checker Methodology ..... 37

3.2.4.1 Linting Strategy ..................................................... 39

3.2.4.2 Implementation Considerations ..................................... 39

3.2.4.3 Event Monitor Database and Analysis ......................... 40

$3.3 \quad$ Summary ......................................................................... 41

\section{RTL Methodology Basics ..............................................43}

4.1 Simple RTL Verifiable Subset ................................................... 44

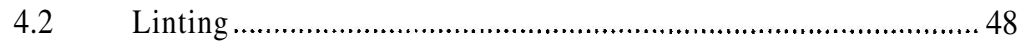

4.2.1 Linting in a design project. ..................................... 49

4.2.2 Lint description ......................................................... 50

4.2.2.1 Project Oriented ........................................................ 50

4.2.2.2 Linting Message Examples............................................ 51

4.3 Object-Based Hardware Design ................................................. 53

4.3.1 OBHD and Simulation................................................. 56

4.3.2 OBHD and Formal Verification ................................. 59

4.3.3 OBHD and Physical Design .......................................... 60

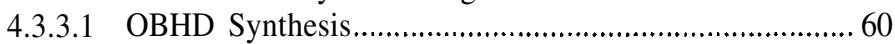

4.3.3.2 OBHD Scan Chain Hookup.......................................... 62

4.3.4 A Text Macro Implementation ...................................... 64

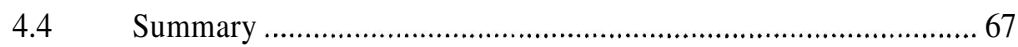

5 RTL Logic Simulation .................................................69

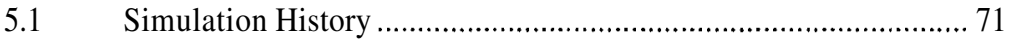

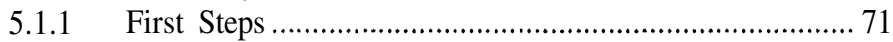




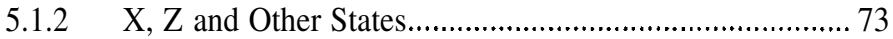

5.1.3 Function and Timing....................................................... 73

5.1.4 Gate to RTL Migration ................................................ 74

5.1.5 Acceleration and Emulation ......................................... 74

5.1.6 Language Standardization ........................................... 76

5.2 Project Simulation Phases .................................................. 78

5.2.1 Debugging Phase ............................................................. 79

5.2.2 Performance Profiling Phase ..................................... 80

5.2.3 Regression Phase ......................................................... 81

5.2.4 Recreating Hardware Problems .................................. 82

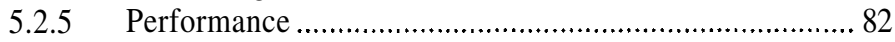

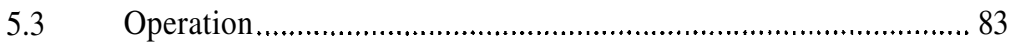

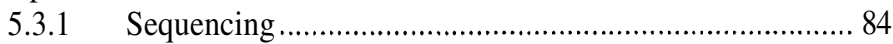

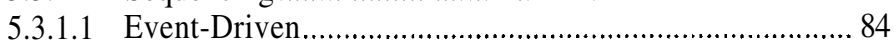

5.3.1.2 Rank-Ordered ..................................................... 86

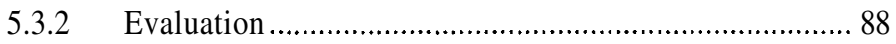

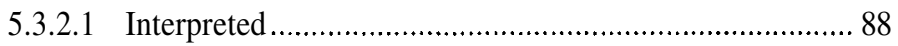

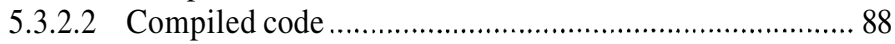

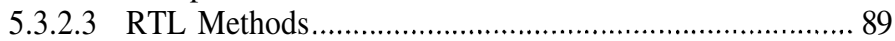

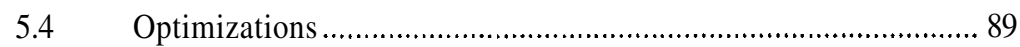

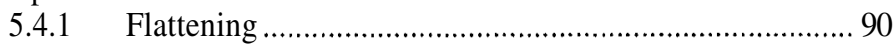

5.4.2 Rank-Ordering .................................................... 90

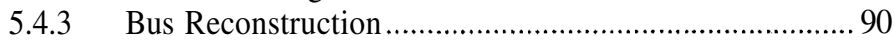

5.4.3.1 Concatenation ........................................................... 91

5.4.3.2 Expression Simplification............................................ 91

5.4.4 OBHD-based Optimization .......................................... 92

5.4.4.1 Common Sub-expression Consolidation ........................ 92

5.4.4.2 Common if-else Control Consolidation.......................... 92

5.4.5 Partitioning .............................................................. 92

5.4.5.1 Branch Partitioning ........................................................ 93

5.4.5.2 Clock Partitioning......................................................... 94

5.4.5.3 Chip Partitioning............................................................ 94

5.5 Random Two-State Simulation Methods ....................................... 95

5.5.1 Start Up State .............................................................. 95

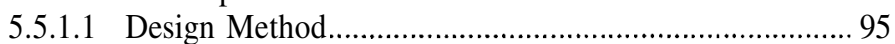

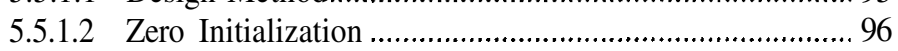

5.5.1.3 Random Initialization ............................................... 97

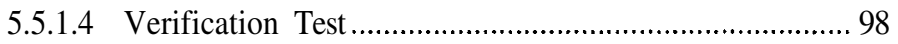

5.5.2 Tri-state Buses ........................................................... 98

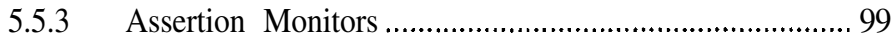

5.5.4 Two-State in the Design Process ................................ 100

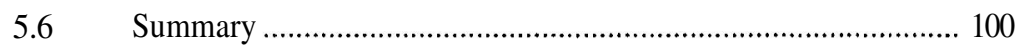


6 RTL Formal Verification ...............................................103

6.1 Formal Verification Introduction............................................... 106

6.2 Finite State Machines............................................................. 107

6.2.1 Machine Equivalence................................................ 110

6.2.2 FSM Property Verification .......................................... 111

6.3 Formal Transformation Verification.......................................... 112

6.3.1 Equivalence Checking ............................................. 112

6.3.1.1 Equivalence Checking Flow ....................................... 112

6.3.1.2 Closing the Verification Loop ..................................... 114

6.3.2 Cutpoint Definition..................................................... 115

6.3.3 Equivalence Checking RTL Coding Style................... 117

6.3.3.1 Isolating Functional Complexity ............................... 117

6.3.3.2 Test Expressions within Case Statements..................... 118

6.3.3.3 Equivalence Checking OBHD Practices .................... 118

6.3.4 RTL Static Sign-off ............................................... 120

6.3.5 Effective Equivalence Checking Methodology ........... 122

6.4 Formal Functional Verification ................................................. 124

6.4.1 Specification .............................................................. 124

6.4.2 Model Checking and Parameterized Modules.............. 126

6.4.3 Model Checking OBHD Practices.............................. 127

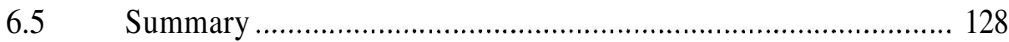

\section{Verifiable RTL Style ......................................................131}

7.1 Design Content......................................................................... 132

7.1.1 Asynchronous Logic ............................................... 132

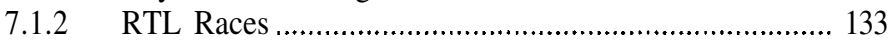

7.1.3 Combinational Feedback .......................................... 135

7.1.4 Case Statements .................................................... 137

7.1.4.1 Fully-Specified case Statements ................................ 138

7.1.4.2 Test Signal and Constant Widths................................. 143

7.1.5 Tri-State Buses........................................................ 144

7.2 Organization.................................................................... 146

7.2.1 System Organization.................................................. 146

7.2.1.1 Compiler Options...................................................... 146

7.2.1.2 Design Hierarchy ...................................................... 148

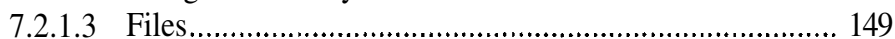

7.2.1.4 Libraries .................................................................. 150

7.2.2 Module Organization ............................................... 151

7.2.2.1 Overall Organization ................................................ 151

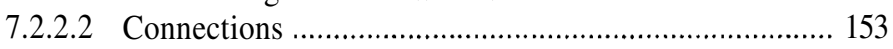

7.2.3 Expression Organization.......................................... 153 
7.3 Naming Conventions............................................................. 155

7.3.1 General Considerations........................................... 155

7.3.1.1 Consistency ......................................................... 155

7.3.1.2 Upper/Lower Case .................................................. 156

7.3.1.3 Hierarchical Name References .................................... 157

7.3.1.4 Global/Local Name Space .......................................... 158

7.3.1.5 Profiling Support ......................................................... 159

7.3.2 Specific Naming Conventions ...................................... 161

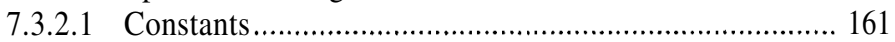

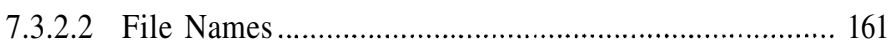

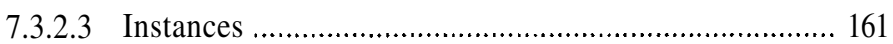

7.3.2.4 Modules ................................................................ 162

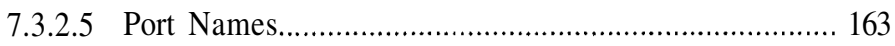

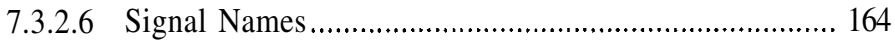

7.3.2.7 User Tasks / Functions and Program Libraries............. 165

7.4 Naming In Verilog Library Modules........................................ 166

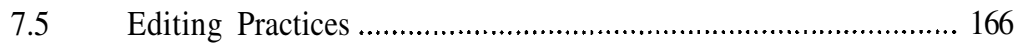

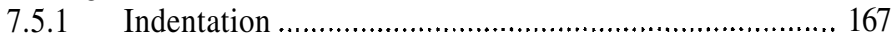

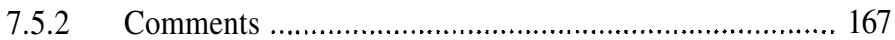

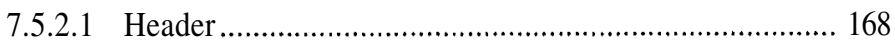

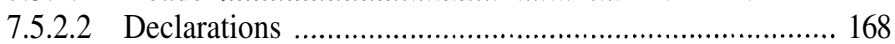

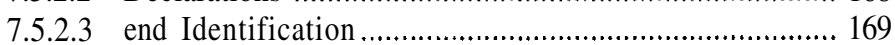

7.5.2.4 Tool Controls ............................................................ 169

7.5.2.5 Embedded Comments ............................................... 171

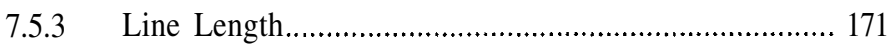

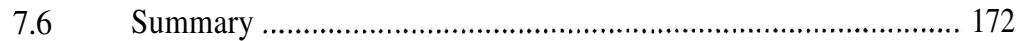

\section{The Bad Stuff ............................................................ 173}

8.1 In-Line Storage Element Specification .................................... 174

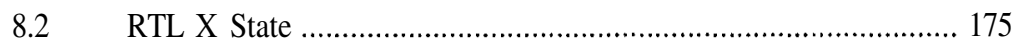

8.2.1 RTL X-STATE PROBLEMS ................................... 176

8.2.1.1 RTL X-State Pessimism .......................................... 176

8.2.1.2 RTL X-State Optimism.............................................. 177

8.2.1.3 Impractical ............................................................. 177

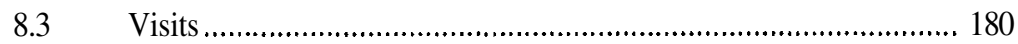

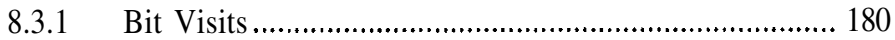

8.3.2 Configuration Test Visits............................................ 181

8.3.3 for Loops................................................................. 182

8.4 Simulation vs. Synthesis Differences........................................ 184

8.4.1 Explicit Differences................................................... 185

8.4.1.1 Full and Parallel Case .................................................... 185

8.4.1.2 X Assignment ......................................................... 188 
8.4.1.3 Other Forms of State Machines ................................... 189

8.4.1.4 Initial blocks ............................................................ 190

8.4.2 Inadvertent Coding Errors ....................................... 191

8.4.2.1 Incomplete Sensitivity List ...................................... 191

8.4.2.2 Latch Inference in functions ..................................... 192

8.4.2.3 Incorrect Procedural Statement Ordering .................... 192

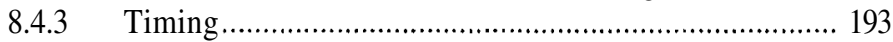

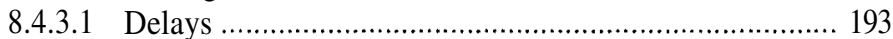

8.4.3.2 Race Conditions ........................................................ 196

8.5 Problematic RTL Verilog ........................................................ 198

8.5.1 Linting and Problematic RTL Verilog........................ 198

8.5.2 Simulation and Problematic RTL Verilog................... 199

8.5.3 Formal Verification and Problematic Verilog ............... 199

8.6 EDA Tool Vendors ............................................................... 200

8.6.1 Tool Library Function Naming ...................................... 201

8.6.2 Command Line Consistency..................................... 202

8.6.3 Vendor Specific Properties .......................................... 203

8.7 Design Team Discipline ......................................................... 204

8.8 Language Elements ............................................................ 205

8.8.1 Keywords ............................................................... 205

8.8.2 Parameters ................................................................. 205

8.8.3 User-Defined Primitives ............................................ 206

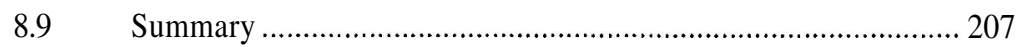

\section{Verifiable RTL Tutorial ...........................................209}

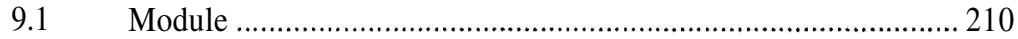

9.1.1 Specification .............................................................. 210

9.1.2 Comments .................................................................. 211

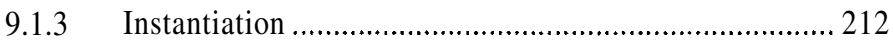

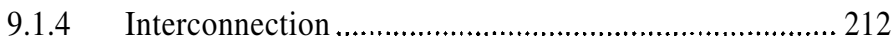

$9.2 \quad$ Adding Behavior ...................................................................... 215

9.3 Multi-bit Interconnect and Behavior ........................................ 216

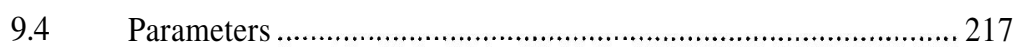

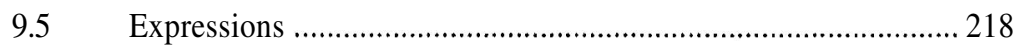

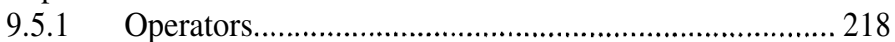

9.5.1.1 Binary operators....................................................... 218

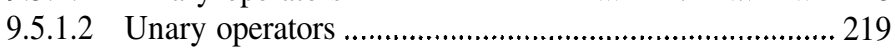

9.5.1.3 Miscellaneous operators .......................................... 220

9.5.2 Operator precedence .................................................. 221

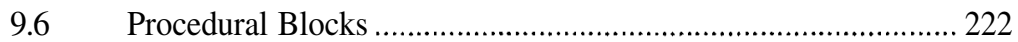

9.6.1 Combinational Logic ................................................ 222 
9.6.1.1 Procedural Assignments ............................................... 222

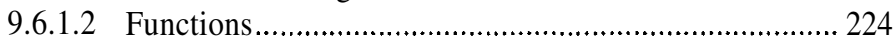

9.6.1.3 if-else Statement ................................................... 225

9.6.1.4 case, casex Statements ............................................ 225

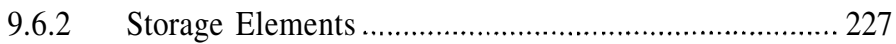

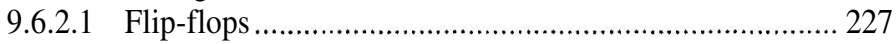

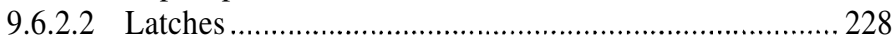

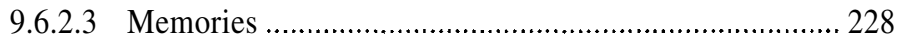

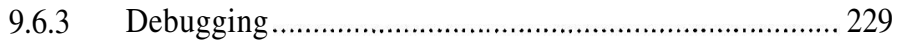

9.6.3.1 \$display and \$write Statements .................................. 230

9.6.3.2 \$finish ................................................................. 231

$9.7 \quad$ Testbench....................................................................... 231

9.8 Verilog Compilation ................................................................. 235

9.8.1 Compiler directives................................................. 235

9.8.1.1 Constants .............................................................. 235

9.8.1.2 Code Inclusion ......................................................... 236

9.8.1.3 Command Line .......................................................... 237

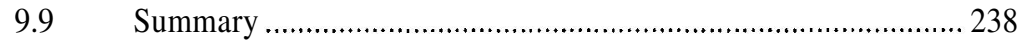

10 Principles of Verifiable RTL Design .........................239

$10.1 \quad$ Principles

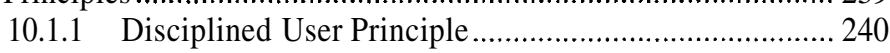

10.1.2 Fundamental Verification Principle............................ 240

10.1.3 Retain Useful Information Principle........................... 240

10.1.4 Orthogonal Verification Principle ............................... 240

10.1.5 Functional Observation Principle ................................. 241

10.1.6 Verifiable Subset Principle ............................................ 241

10.1.7 Project Linting Principle............................................... 241

10.1.8 Object-Based Hardware Design Principle .................... 242

10.1.9 Fast Simulation Principle ............................................. 242

10.1.10 Visit Minimization Principle ........................................ 242

10.1.11 Cutpoint Identification Principle ................................. 242

10.1.12 Numeric Value Parameterization Principle .................. 243

10.1.13 Consistency Principle ................................................. 243

10.1.14 Asynchronous Principle ............................................... 244

10.1.15 Combinational Feedback Principle ................................ 244

10.1.16 Property Principle ........................................................ 244

10.1.17 Faithful Semantics Principle........................................ 244

10.1.18 Good Vendor Principle .................................................. 245

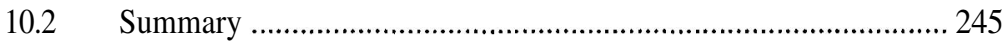


A. Comparing Verilog Construct Performance .............255

B. Quick Reference ...................................................................259

C. Assertion Monitors .....................................................265 


\section{FOREWORD}

System designers, computer scientists and engineers have continuously invented and employed notations for modeling, specifying, simulating, documenting, communicating, teaching, verifying and controlling the designs of digital systems. Initially these systems were represented via electronic and fabrication details. Following C. E. Shannon's revelation of 1948, logic diagrams and Boolean equations were used to represent digital systems in a fashion that de-emphasized electronic and fabrication detail while revealing logical behavior. A small number of circuits were made available to remove the abstraction of these representations when it was desirable to do so. As system complexity grew, block diagrams, timing charts, sequence charts, and other graphic and symbolic notations were found to be useful in summarizing the gross features of a system and describing how it operated. In addition, it always seemed necessary or appropriate to augment these documents with lengthy verbal descriptions in a natural language.

While each notation was, and still is, a perfectly valid means of expressing a design, lack of standardization, conciseness, and formal definitions interfered with communication and the understanding between groups of people using different notations. This problem was recognized early and formal languages began to evolve in the 1950s when I. S. Reed discovered that flip-flop input equations were equivalent to a register transfer equation, and that 
tor-like notation. Expanding these concepts Reed developed a notation that became known as a Register Transfer Language (RTL). While the notation had only a few features that are associated with RTLs today, its development started an evolutionary process that is still underway. RTLs such as Verilog improve the ability of the designer to communicate the design intent as well as providing a mechanism to verify correctness.

In the late 1980s, progress in RTL synthesis provided productivity gains by translating the designer's intent into gates and silicon. Success with RTL synthesis resulted in the acceptance of an RTL synthesizable subset. In other words, by constraining a rich RTL to a subset of keywords and an explicit coding style, synthesis can use precise semantics to provide an efficient and clear mapping to a specific hardware implementation.

Unfortunately, the synthesis productivity gain has resulted in an increase in verification complexity. In this book, the focus is on verification productivity gains. This seems to be a normal progression to emphasize synthesis productivity gains when synthesis was in the critical path, and then turn to verification productivity gains when it became the biggest problem for the design team. This book promotes a strong coupling between the RTL specification and the verification process. The authors have placed emphasis on describing verification processes and explicitly how they relate to RTL coding style. Many other Verilog books describe details for the entire language without considering the coding impact related to the verification process.

By constraining a rich hardware description language to an RTL verifiable subset, emerging verification technologies can easily be integrated into the design flow (e.g., model checking, equivalence checking, cycle-based simulation and fast two-state simulation). The RTL verifiable subset in this book is actually a smaller synthesizable subset, which includes the keywords supporting higher levels of modeling and abstraction. Some Hewlett-Packard engineers outside the authors' team have found the ideas too revolutionary, and out-of-line with the way that they learned to use Verilog for simulation and synthesis. However, the authors' team has successfully developed very large complex systems in Hewlett-Packard following the guidelines and methodologies discussed in this book. Furthermore, the authors' team has successfully integrated emerging verification technologies into their design flow, while other design teams are struggling and fighting the verification tools and processes.

I find this book to be a significant contribution in helping other 
teams of engineers cope with large system design complexity and survive the verification processes, which have become the most time consuming critical path item in the design process today.

James R. Duley, Ph.D.

Hewlett-Packard Company

Palo Alto. California 


\section{PREFACE}

The need for a verifiable RTL philosophy is justified by the complexity, density, and clock speeds of today's chips and systems.
The conception of a verifiable register transfer level (RTL) philosophy is a product of two factors: one, inherited seat-of-the-pants experiences during the course of large system design; the other, the sort of investigation which may be called "scientific." Our philosophy falls somewhere between the knowledge gained through experiences and the knowledge gained through scientific research. It corroborates on matters as to which definite knowledge has, so far, been ascertained; but like science, it appeals to reason rather than authority. Our philosophy consists of a fundamental set of principles, which when embraced, yield significant pay back during the process of verification.

The need for a verifiable RTL philosophy is justified by the complexity, density, and clock speeds of today's chips and systems, which continue to grow at exponential rates. This situation has raised the cost of design errors to a critical point--where, increasingly, the resources spent on the process of verification exceeds those spent on design.

Myriad books, manuals, and articles have addressed the issue of RTL Verilog style with an emphasis on synthesis-oriented policies. They explain how to write Verilog to wrest optimal gates from the synthesis process. Still other material presents the entire spectrum of Verilog constructs from the architectural specification to 
We have
deliberately
linked the
RT-level
verification
process to the
language and
have chosen
not to discuss
the details of
the Verilog
language
reference
manual.

Intended

Audience. switch-level strengths. Yet, these works leave it to the readers to find their way to good practices for verification. Unfortunately, few guidelines govern the coding of RTL Verilog to achieve an optimum flow through the various functional and logical verification processes.

This vacuum clearly becomes a problem as design complexity increases, and as design teams consider incorporating more advanced traditional and formal verification processes within their flow (for instance, cycle-based simulation, two-state simulation, model checking and equivalence checking). Our solution is to introduce a verifiable subset of Verilog and a simple RTL coding style. The coding policies we present have enabled us to effectively incorporate these new verification technologies into our design flow. To provide a framework for discussion, we place emphasis on describing verification processes throughout the text--as opposed to an in-depth discussion of the Verilog language. Specifically, we are interested in how an engineer's decision to code their RTL impacts a verification tool's performance and the quality of the overall verification process. Thus, we have deliberately linked the RT-level verification process to the language and have chosen not to discuss the details of the Verilog language reference manual.

In writing and public speaking training, students are always told to know their reader and audience, and adjust their presentation accordingly. In verification, the audience for a design description is the verification processes and tools. This book presents the verification process and tools in the first chapters, then presents RTL Verilog in later chapters.

This book tells how you can write Verilog to describe chip designs at the RT-level in a manner that cooperates with verification processes. This cooperation can return an order of magnitude improvement in performance and capacity from tools such as simulation and equivalence checkers. It reduces the labor costs of coverage and formal model checking, by facilitating communication between the design engineer and the verification engineer. It also orients the RTL style to provide more useful results from the overall verification process.

One intended audience for this book is engineers and students who need an introduction to various design verification processes and a supporting functional Verilog RTL coding style. A second intended audience is engineers who have been through introductory training in Verilog, and now want to develop good RTL writing practices for verification. A third audience is Verilog language instructors who are using a general text on Verilog as the course 
textbook, but want to enrich their lectures with an emphasis on verification. A fourth audience is engineers with substantial Verilog experience who want to improve their Verilog practice to work better with RTL Verilog verification tools. A fifth audience is design consultants searching for proven verification-centric methodologies. A sixth audience is EDA verification tool implementers who want some suggestions about a minimal Verilog verification subset.

The concepts presented in this book are drawn from the authors' experience with large-scale system design projects. The scale of these design projects ranged to more than 200 million gate-equivalents, and we are happy to report that the products were commercially successful. To support the design processes in these projects, we evaluated and integrated verification tools from the marketplace. Before there were commercially available tools, we developed the tools ourselves. The tools include equivalence checkers, cycle-based simulators, linters, implicit interconnection, macro preprocessors, and RTL scan simulation support.

\section{Second Edition}

The first edition of Principles of Verifiable RTL Design offered a common sense method for simplifying and unifying assertion specification. This method includes creating a set of predefined specification modules that can be instantiated within the designer's RTL.

Open

Verification

Library

Initiative.

Since the release of the first edition, an industry wide initiative for assertion specification has emerged based on ideas presented in our book. This initiative, known as Open Verification Library Initiative (www.verificationlib.org), provides an assertion interface standard that allows the design engineer to capture many interesting properties of the design and precludes the need to introduce new HDL constructs (i.e., extensions to Verilog are not required). Furthermore, this standard enables the design engineer to "specify once," then target the same RTL assertion specification over multiple verification processes, such as traditional simulation, semi-formal and formal verification tools. We view the Open Verification Library Initiative as an empowering technology that will benefit design and verification engineers, and establish unity among the EDA community (i.e., providers of testbench generation tools, traditional simulators, commercial assertion checking support tools, symbolic simulation, and semi-formal and formal verification tools). We are delighted that our book has provided a positive influenced on both design teams and the EDA industry. 
Expanded discussion.

Reorganized topic presentation.

21 principles combined into 18.

Two principles change their wording.

Superlog and SystemC.

\section{Keeping verification current.}

Visit our web site.
This second edition of Principles of Verification RTL Design expands the discussion of assertion specification by including a new chapter, titled Coverage, Events and Assertions. All assertions exampled are aligned with the Open Verification Library Initiative proposed standard. In addition, the second edition provides expanded discussions on the following topics:

- start-up verification

- the place for 4-state simulation

- race conditions

- RTL-style - synthesizable RTL. (unambiguous mapping to gates)

- more "bad stuff"

Since the first edition, we have presented tutorials on Verifiable RTL Design in dozens of venues to a total of over 2,000 design engineers. Based on their feedback, we have tuned up the order of presentation in many chapters, but particularly in Chapter 4.

Of the 21 principles from the first edition, 15 remain intact. The changes in principles in the second edition include:

- folding the Test Expression Observability Principle into the Cutpoint Identification Principle,

- combining the Indentation Principle, the Code Inclusion Control Principle, and the Entry Point Naming Principle into a single Consistency Principle, and

- changing the Meta-comment Principle into the Property Principle, and the Object-Oriented Hardware Design Principle into the Object-Based Hardware Design Principle.

At the time of this writing, verification processes and tools for Superlog and SystemC are not ready. Without design project experience, RTL verification based on these languages could only be projections.

Our goal in releasing a second edition is to keep the topic current. The bibliography has been expanded to include the latest research. Every chapter of the book has been enhanced based on positive and critical feedback. Finally, the overall book's look and feel has been enhanced to a more contemporary appearance.

Readers can find more information about this book and e-mail the authors from the URL www.verifiableRTL.com. 


\section{Acknowledgments}

The authors wish to thank the following people who participated in discussions, made suggestions and other contributions to our Principles of Verifiable RTL Design project:

Greg Brinson, Bill Bryg, Christian Cabal, Dr. Albert Camilleri, Dino Caporossi, Michael Chang, Dr. K.C. Chen, Dr Kwang-Ting (Tim) Cheng, Carina Chiang, Dr. Claudionor Coelho, Dr. James R. Duley, Jeanne Foster, Bryan Hornung, Michael Howard, Tony Jones, James Kim, Ruth McGuffey, Dr. Gerard Memmi, Dr. Ratan Nalumasu, Bob Pflederer, Dr. Carl Pixley, Dr. Shyam Pullela, Rob Porter, David Price, Hanson Quan, Jeff Quigley, Mark Shaw, Dr. Eugene Shragowitz, Dr. Vigyan Singhal, Bob Sussman, Paul Vogel, Ray Voith, Chris Yih, Nathan Zelle, and numerous design engineers from the Hewlett-Packard Richardson Lab. 